\title{
Lacunary Fourier series and a qualitative uncertainty principle for compact Lie groups
}

\author{
E K NARAYANAN and A SITARAM \\ Department of Mathematics, Indian Institute of Science, Bangalore 560 012, India \\ E-mail: naru@math.iisc.ernet.in; sitaram.alladi@gmail.com
}

MS received 19 July 2010; revised 24 November 2010

\begin{abstract}
We define lacunary Fourier series on a compact connected semisimple Lie group $G$. If $f \in L^{1}(G)$ has lacunary Fourier series and $f$ vanishes on a non empty open subset of $G$, then we prove that $f$ vanishes identically. This result can be viewed as a qualitative uncertainty principle.
\end{abstract}

Keywords. Lacunary Fourier series; uncertainty principles; Weyl's character formula.

\section{Introduction}

A set $A \subset \mathbb{Z}$ is called $Q$-thin if it is contained in $\mathbb{N}$ or $-\mathbb{N}$ and if

$$
|n| /|m| \geq Q \forall n, m \in A,|n|>|m| .
$$

The set $A$ is called lacunary (in the sense of Hadamard (see page 109 of [2])) if, either $A$ is empty or there are $N \in \mathbb{N}$ and $Q>1$ such that $A \cap[N, \infty)$ and $A \cap(-\infty,-N]$ are $Q$-thin. Then the following result is well known.

Theorem 1.1. Let $A$ be a finite union of lacunary sets and $g \in L^{2}(\mathbb{T})$ be such that $\{m: \hat{g}(m) \neq 0\} \subset A$. Then $g$ vanishes identically provided $g$ vanishes on a set of positive measure.

This can be proved using the fact that finite union of lacunary sets is sparse. See page 109-110 of [2]. See also Chapter V, Theorem 6.13 of [9].

Our aim in this paper is to define lacunarity on a compact connected semisimple Lie group $G$, using the canonical parametrization of irreducible unitary representations of $G$. Using our definition, we prove that, if $f \in L^{1}(G)$ has lacunary Fourier series and $f$ vanishes on a non empty open subset of $G$, then $f$ vanishes identically. Before we state and prove our main result, let us recall some facts.

Let $G$ be a compact connected semisimple Lie group and $T$ a maximal torus of $G$. The Lie algebras of $G$ and $T$ will be denoted by $\mathfrak{g}$ and $\mathfrak{t}$, respectively. Let $\mathfrak{g} \mathbb{C}$ and $\mathfrak{t}_{\mathbb{C}}$ denote the complexifications of $\mathfrak{g}$ and $\mathfrak{t}$ respectively.

We now describe the Weyl character formula for $G$ which will be needed later. For any unexplained terminology, we refer to [8]. 
Theorem 1.2 Let $G$ be a compact connected semisimple Lie group and let $R^{+}$be a positive system of roots. Then the irreducible characters of $G$ are in one-to-one correspondence with the positive characters of $T$ (whose derivatives satisfy conditions (I) and (II) listed below). If $\chi$ is one such character of $T$, the corresponding irreducible character $\Theta_{\chi}$ of $G$ (restricted to $T$ ) is given by

$$
\Theta_{\chi}=\left(\sum_{w} \varepsilon(w) \chi^{w}\right) / \Delta^{+},
$$

where $\Delta^{+}=\sum_{w} \varepsilon(w) \xi_{\rho}^{w}$ (see page 40 of $[8]$ ).

Here $\rho$ is the half-sum of positive roots and $\xi_{\rho}^{w}=w \cdot \xi_{\rho}$ where $\xi_{\rho}(\exp H)=\mathrm{e}^{\rho(H)}$ and $w \cdot \chi$ is the action of the Weyl group on the characters $\chi$ of $T$. Notice that $\Delta^{+}(t) \Theta_{\chi}(t)$ is a finite Fourier series on $T$.

The unitary dual of $G$, denoted by $\hat{G}$, is described by the theorem of highest weight and is parametrized by a certain lattice in $\mathfrak{t}_{\mathbb{C}}^{*}$. If $\lambda \in \mathfrak{t}_{\mathbb{C}^{*}}$, then $\lambda$ is a highest weight and gives rise to a unitary irreducible representation of $G$ if and only if the following two conditions are satisfied:

(I) Positivity and integrality conditions: $\frac{2 \lambda\left(H_{\alpha}\right)}{\alpha\left(H_{\alpha}\right)} \in \mathbb{Z}^{+}$for all $\alpha \in R^{+}$, the set of positive roots,

(II) $\lambda$ arises from a character of $T$, i.e. $\lambda$ restricted to $t$ is the derivative of a character on $T$.

It follows that $\hat{G}$ can be identified with a subset of a (full) lattice contained in the dual of $T$. For more on compact semsimiple Lie groups, we refer to [5] and [8].

Next, we define lacunary Fourier series on $G$. Let $f$ be an $L^{1}$ function on $G$ and let $\pi(f)$ denote the Fourier transform of $f$ defined by

$$
\pi(f)=\int_{G} f(x) \pi(x) \mathrm{d} x,
$$

where $\pi$ is an irreducible unitary representation of $G$. As mentioned earlier $\hat{G}$ may be identified with a subset of a lattice in the dual of the maximal torus $T$. This is done as follows: We identify the lattice generated by the elements in $\mathfrak{t}_{\mathbb{C}}^{*}$ satisfying (I) and (II) above with a subset of the lattice $\mathbb{Z}^{k}$ where $k=\operatorname{dim} T$. We say that $f \in L^{1}(G)$ has lacunary Fourier series if the set $E=\{\pi: \pi(f) \neq 0\}$ (considered as the lattice defined earlier) satisfies the following:

$$
\bigcup_{w \in W} w \cdot E \subset E_{1} \times E_{2} \times \cdots \times E_{k},
$$

where each $E_{j} \subset \mathbb{Z}, j=1,2, \ldots, k$ is a finite union of lacunary sets. We emphasize that our definition of lacunarity differs from that of Helgason (see [3]).

\section{Proof of the main result}

In this section we prove the main result. We start with the following lemma. 
Lemma 2.1. Let $g \in C\left(\mathbb{T}^{k}\right)$ and $\operatorname{spec}(g)=\left\{\hat{g}(\mathbf{m}) \neq 0: \mathbf{m} \in \mathbb{Z}^{k}\right\} \subset E_{1} \times E_{2} \times \cdots \times E_{k}$, where each $E_{j} \subset \mathbb{Z}, j=1,2, \ldots, k$ is a finite union of lacunary sets. If $g$ vanishes on a non empty open subset of $\mathbb{T}^{k}$, then $g$ vanishes identically.

Proof. This follows from Theorem 1.1 and induction on $k$.

Now we are in a position to state and prove the main result of this paper.

Theorem 2.2. Suppose $f \in L^{1}(G)$ has lacunary Fourier series. If $f$ vanishes on a non empty open subset of $G$, then $f \equiv 0$.

Proof. Let $\operatorname{spec}(f) \subset \mathbb{Z}^{k}$ correspond to $\{\pi \in \hat{G}: \pi(f) \neq 0\}$. Notice that if $f$ has lacunary Fourier series then so do the translates of $f$. Hence, without loss of generality we may assume that $f$ vanishes in a neighborhood of the identity in $G$. Convolving $f$ with smooth approximate identities supported in small neighborhoods of the identity of $G$, we may assume that $f$ is continuous, indeed smooth.

Now, consider the function $F_{f}$ defined as

$$
F_{f}(x)=\int_{G} f\left(g x g^{-1}\right) \mathrm{d} g .
$$

Since one can choose a metric on $G$ which is biinvariant, it is clear that $F_{f}$ vanishes in a small enough neighborhood of identity in $G$. Also, $F_{f}$ is a central function and so is determined by its restriction (still denoted by $F_{f}$ ) to the maximal torus $T$. A simple computation using Schur's orthogonality relations shows that

$$
F_{f}(t)=\sum_{\pi \in \hat{G}} \operatorname{Trace}(\pi(f)) \Theta_{\pi}(t),
$$

where $t \in T$ and $\Theta_{\pi}$ is the character of $\pi$ defined by $\Theta_{\pi}(x)=\operatorname{Trace}(\pi(x))$. By Weyl's character formula we get

$$
\Delta^{+}(t) F_{f}(t)=\sum_{\pi \in \hat{G}} \operatorname{Trace}(\pi(f))\left(\sum_{w} \varepsilon(w) \chi_{\pi}^{w}(t)\right),
$$

where the positive character $\chi_{\pi}$ of $T$ corresponds to $\pi \in \hat{G}$. Notice that, the set of nonzero Fourier coefficients of $\Delta^{+}(t) F_{f}(t)$ is contained in $\bigcup_{w \in W} w \cdot \operatorname{spec}(f)$ which satisfies eq. (1.1). Since $F_{f}$ vanishes on an open set, by Lemma $2.1, F_{f}$ vanishes identically. It follows that $\operatorname{Trace}(\pi(f))$ is zero for $\pi \in \hat{G}$.

Next, notice that any translate of $f$ has lacunary Fourier series. If $g$ varies in a small enough neighborhood of the identity in $G$, then applying the above argument to the translated function ${ }^{g} f(x)=f(g x)$ we obtain that Trace $\left(\pi\left(g^{-1}\right) \pi(f)\right)$ is zero for all $g$ in a small neighborhood and all $\pi \in \hat{G}$. For each $\pi, g \rightarrow \operatorname{Trace}\left(\pi\left(g^{-1}\right) \pi(f)\right)$ is a real analytic function and hence it follows that $\operatorname{Trace}\left(\pi\left(g^{-1}\right) \pi(f)\right)$ is identically zero for each $\pi$. This clearly implies that $f$ is zero, by the inversion formula. 
Remark 2.3. Using the parametrization of the unitary dual of a compact connected Lie group $G$ via the highest weight theorem, one may extend the main result to compact groups, without the assumption of semisimplicity.

Remark 2.4. If $G=U(n)$, then $\hat{G}$ can be canonically identified with a subset of $\mathbb{Z}^{n}$ (see [8]). The Weyl group $W$ is the permutation group $S_{n}$. If $A=A_{1} \times A_{2} \times \cdots \times A_{n}$ where each $A_{j} \subset \mathbb{Z}, j=1,2, \ldots, n$ is lacunary. Then it is easy to see that $A$ satisfies eq. (1.1). (Note that $U(n)$ is not semisimple.) We feel that even in the general case, the main theorem will go through with this more relaxed definition of lacunarity. However, at the moment we are not able to prove this.

Remark 2.5. Ideally one would like to get the exact analogue of Theorem 1.1, but the averaging method used here to convert the problem to one on the torus works only if we assume that the set on which $f$ vanishes is open. If $f$ vanishes on a set of positive measure, then $F_{f}$ considered as a function on $\mathbb{T}$ need not necessarily vanish on a set of positive measure on $\mathbb{T}$. However, if $f$ vanishes on a neighborhood of the identity in $G$, then $F_{f}$ considered as a function on $\mathbb{T}$ vanishes on a neighborhood of the identity in $\mathbb{T}$.

\section{Uncertainty principles}

To quote G B Folland, "the uncertainty principle is a meta theorem in harmonic analysis that can be summarized as follows: A non zero function and its Fourier transform cannot both be sharply localized." For more on uncertainty principles on compact groups, we refer to [7] (see also Theorem 2.6 in [4]) and [6] and for uncertainty principles, in general, to $[1]$.

A function on $\mathbb{Z}^{n}$ can be thought of as localized, if it is supported on a lacunary set. The opposite of a localized function is a spread out function. A function whose closed support is all of $G$ can be thought of as a highly spread out function. Thus Theorem 2.2 says that if the Fourier transform of a function on $G$ is localized, then the function itself has to be necessarily spread out - thus illustrating the uncertainty principle in the context of compact groups.

\section{Acknowledgement}

We thank G B Folland for drawing our attention to Chapter V, Theorem 6.13 of [9]. The first-named author was supported in part by a grant from UGC via DSA-SAP and the second-named author was supported by the Raja Ramanna Fellowship of DAE.

\section{References}

[1] Folland G B and Sitaram A, The uncertainty principle: a mathematical survey, J. Fourier Anal. Appl. 3(3) (1997) 207-238

[2] Havin V and Jöricke B, The uncertainty principle in harmonic analysis (Springer-Verlag) (1994)

[3] Helgason S, Lacunary Fourier series on noncommutative groups, Proc. Am. Math. Soc. 9 (1958) 782-790 
[4] Hogan J A, A qualitative uncertainty principle for unimodular groups of type I, Trans. Am. Math. Soc. 340(2) (1993) 587-594

[5] Knapp A W, Representation theory of semisimple Lie groups. An overview based on examples (Princeton, NJ: Princeton University Press) (2001)

[6] Kutyniok G, A weak qualitative uncertainty principle for compact groups, Ill. J. Math. 47(3) (2003) 709-724

[7] Price J F and Sitaram A, Local uncertainty inequalities for compact groups, Proc. Am. Math. Soc. 103(2) (1988) 441-447

[8] Varadarajan V S, An introduction to harmonic analysis on semisimple Lie groups, Cambridge Studies in Advanced Mathematics, 16 (Cambridge: Cambridge University Press) (1989)

[9] Zygmund A, Trigonometric series, vol I, 2nd edn (Cambridge University Press) (1959) 